\title{
Correlation Between Left Ventricular Diastolic Function and Ejection Fraction in Dilated Cardiomyopathy Using Magnetic Resonance Imaging With Late Gadolinium Enhancement
}

\author{
Shuji Nanjo, MD; Kohki Yoshikawa, MD*; Masahiko Harada, MD**; Yusuke Inoue, MD ${ }^{\dagger}$; \\ Atsushi Namiki, MD; Hajime Nakano, MD; Junichi Yamazaki, MD
}

\begin{abstract}
Background: The distribution of left ventricular (LV) fibrosis and the percent fibrosis in patients with dilated cardiomyopathy (DCM) were evaluated using late gadolinium enhanced (LGE) MRI. Then the relation with the LV ejection fraction (EF) and deceleration time (DT), an index of diastolic function obtained using echocardiography, was investigated.

Methods and Results: LGEMRI at $20 \mathrm{~min}$ after intravenous injection of Gd-DTPA $(0.15 \pm 0.03 \mathrm{mmol} / \mathrm{kg})$ was performed in 17 patients with DCM. The distribution of the LV enhanced area and LGE rate (\%) were calculated. EF, as well as E/A ratio and DT were obtained using echocardiography. LGE was observed in 15 out of 17 patients (88\%) and the enhanced region appeared to represent myocardial fibrosis. The LV fibrosis was often found in the intraventricular septum (IVS), but there were no differences in its distribution. The LGE rate (\%) had a correlation between cardiac magnetic resonance ejection fraction (CMREF) $\left(\mathrm{Y}=51.7-2.1 \mathrm{X}\left[\mathrm{R}^{2}=0.23, \mathrm{P}<\right.\right.$ $0.001])$ and $\mathrm{DT}\left(\mathrm{Y}=162.2+12.0 \mathrm{X}\left[\mathrm{R}^{2}=0.35, \mathrm{P}<0.001\right]\right)$.

Conclusions: The LV fibrosis is often found in the IVS with DCM. A correlation exists between LGE rate (\%) to EF on CMR and DT on echocardiography in patients with DCM. (Circ J 2009; 73: 1939-1944)
\end{abstract}

Key Words: Cardiac magnetic resonance imaging; Cardiomyopathy; Diastolic function

I n 1985, Farmer et al reported that the signal intensity (SI) increased during magnetic resonance imaging (MRI) of myocardial lesions in patients with hypertrophic cardiomyopathy (HCM) after Gd-DTPA (gadolinium diethylenetriamine pentaacetic acid) was administered as a contrast medium. ${ }^{1}$ There have been some reports that myocardial lesions can be evaluated from their enhancement by Gd-DTPA on T1-weighted images. ${ }^{2,3}$

It was also reported that the region of myocardial fibrosis is demonstrated by late gadolinium enhanced imaging (LGE), which is performed at $20 \mathrm{~min}$ after Gd-DTPA inversion recovery (IR) imaging. ${ }^{4,5}$ In recent years, cardiac MRI findings have been investigated in patients with idiopathic dilated cardiomyopathy (DCM) and relatively thin ventricular walls. ${ }^{6-8}$ The present study investigated the patterns and rates of LGE by cardiac MRI in DCM, as well as the correlation between myocardial fibrosis and left ventricular (LV) diastolic dysfunction shown by echocardiography or cardiac magnetic resonance (CMR). ${ }^{9-11}$

\section{Methods}

\section{Subjects}

The subjects of the present study were 17 patients ( 15 males and 2 females aged $52 \pm 4$ years) who were diagnosed as having DCM according to the 1996 WHO diagnostic criteria in 1996. ${ }^{12}$ These 17 patients were in New York Heart Association class II $(n=16)$ or class III $(n=1)$. DCM was diagnosed based on exclusion of other causes of LV dysfunction, such as acute myocarditis, significant coronary artery stenosis, valvular disease and/or other secondary myocardial diseases. The patients were treated with $\beta$-blockers (83\%), angiotensin-converting enzyme inhibitors (ACE-I) (39\%) and/or angiotensin II receptor blockers (ARB) (39\%).

\section{Cine MRI and LEG Images}

Cine MRI was performed between the cardiac base and apex in the LV short axis view and LEG images were obtained at $20 \mathrm{~min}$ after Gd-DTPA was administered. Then the LV imaging patterns and LGE rate were calculated. 13,14 Cardiac systolic function and LV diastolic function were evaluated using echocardiography performed within 2 weeks of the cardiac MRI study.

The device used for MRI was a $1.5 \mathrm{~T}$-scanner (Signa CV/i, GE Medical System, Milwaukee, WI, USA). After the position was decided and Gd-DTPA $(0.15 \pm 0.03 \mathrm{mmol} / \mathrm{kg})$ was injected intravenously, cine MRI was done and LGE imaging performed $20 \mathrm{~min}$ after administration.

(Received October 15, 2008; revised manuscript received May 21, 2009; accepted June 14, 2009; released online September 4, 2009)

Division of Cardiovascular Medicine, Department of Internal Medicine, Toho University Omori Medical Center, *Department of Radiotechnical Sciences, Facutly of Health Sciences, Komazawa University, **Department of Clinical Functonal Physiology, Toho University Omori Medical Center and †Department of Radiology, Institute of Medical Sciences, University of Tokyo, Tokyo, Japan

Mailing address: Shuji Nanjo, MD, Division of Cardiovascular Medicine, Department of Internal Medicine, Toho University Omori Medical Center, 6-11-1 Omori-nishi, Ota-ku, Tokyo 143-8541, Japan. E-mail: maru@pd5.so-net.ne.jp

All rights are reserved to the Japanese Circulation Society. For permissions, please e-mail: cj@j-circ.or.jp 


\section{Slices of LV using LGE}

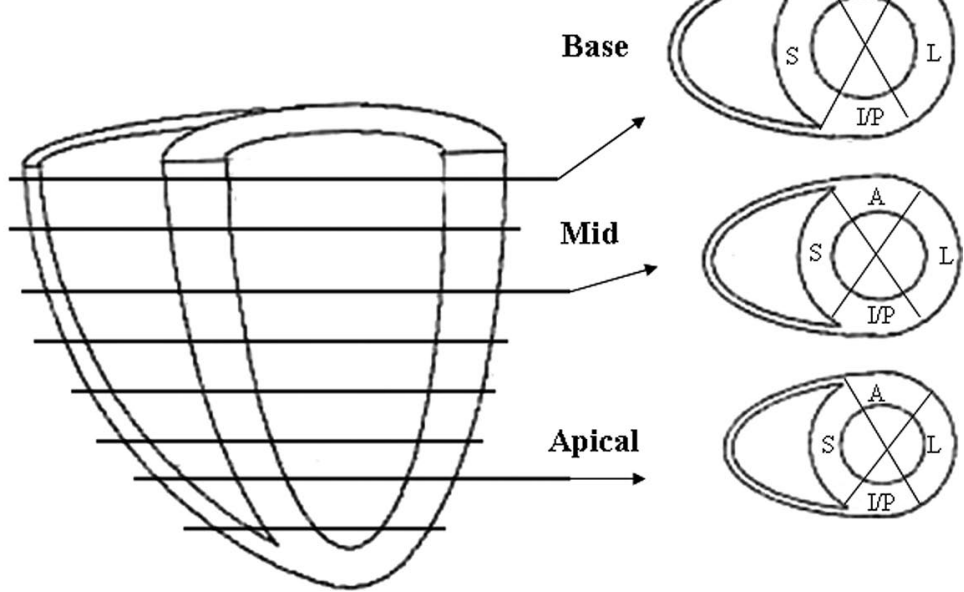

a

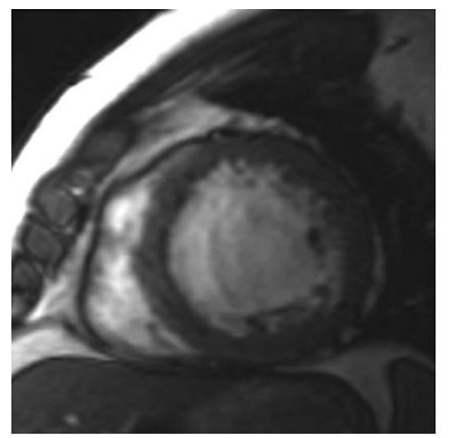

C

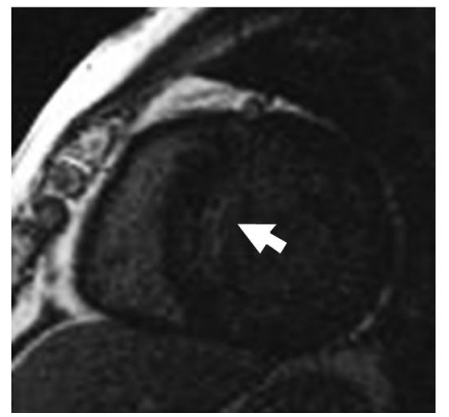

b

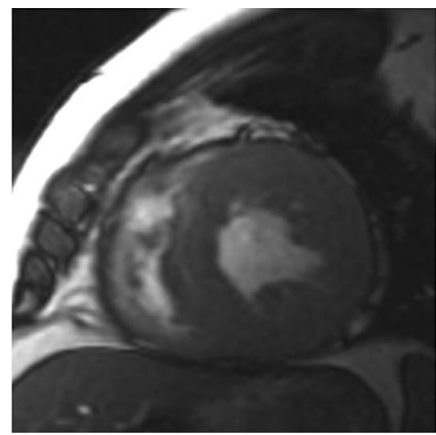

d

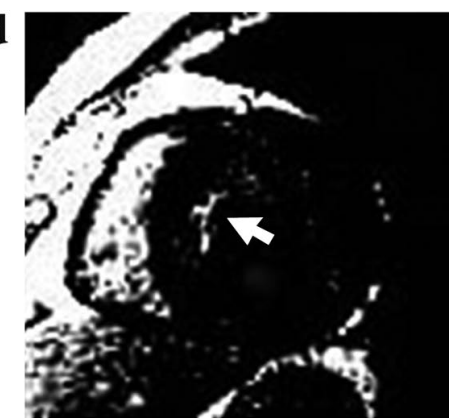

Figure 1. Standardized myocardial segmentation for cardiac imaging. Each slice was divided into segments of the anterior wall (A), lateral wall (L), inferior/posterior wall (I/P) and septum wall (S). LV, left ventricular; LGE, late gadolinium enhanced imaging.

Figure 2. (a) Image was a left ventricular (LV) short axis cine image of the end-diastolic phase. The wall thickness was less than $10 \mathrm{~mm}$ and $\mathrm{LV}$ diastolic diameter was $64 \mathrm{~mm}$, LV dilatation. (b) Image was a cine image of the end-systolic phase. LV wall motion was improved by $\beta$-blockers and ARB. (c) Image was a LV short axis image and the septum of LV was enhanced using enhanced CMR. (d) Image of scion analysis image. We set threshold and calculated an enhanced area (pixel) visually using enhanced CMR.
Table. Characteriatics of the Study Patients

\begin{tabular}{lc}
\hline Number $(\mathrm{M} / \mathrm{F})$ & $17(15 / 2)$ \\
Age & $52 \pm 4$ \\
New York Heart Association II/III & $16 / 1$ \\
Medication & \\
$\quad \beta$-blocker & $15(83 \%)$ \\
ACE-I/ARB & $9 / 7(89 \%)$ \\
CMR & \\
LV mass (g) & $135.0 \pm 40.0$ \\
CMREF $(\%)$ & $43.8 \pm 16.6^{*}$ \\
LVEDV $\left(\mathrm{cm}^{3}\right)$ & $169.5 \pm 55.0^{\dagger}$ \\
LVESV $\left(\mathrm{cm}^{3}\right)$ & $93.2 \pm 46.2$ \\
LGE rate $(\%)$ & $3.84 \pm 3.9$ \\
Echocardiography & \\
Echo EF $(\%)$ & $51.9 \pm 13.8^{*}$ \\
LVDd (mm) & $62.9 \pm 8.2^{\dagger}$ \\
E/A & $1.15 \pm 0.6$ \\
DT (ms) & $208.4 \pm 78.1$ \\
\hline
\end{tabular}

$(*,+: \mathrm{P}<0.001)$ values are mean $\pm \mathrm{SD}$.

Abbreviations see in text.
Cine MRI images were obtained for 6-8 slices of the area between the LV base and the apex using the fast gradient field echo technique (GE: TR $=7.1 \mathrm{~ms}, \mathrm{TE}=3.1 \mathrm{~ms}$, Flip angle $60^{\circ}$, FOV $38 \times 38 \mathrm{~cm}$, Matrix $256 \times 192$, Slice Thickness $=9 \mathrm{~mm}$, Gap $1 \mathrm{~mm}$ ) and in synchronization with the electrocardiogram while patients held their breath. Cine MRI data on the area between the LV base and apex were transmitted to Advantage Wind (version 5.0) software on a work station (GE Yokogawa Medical System) for analysis with cardiac and flow analysis tools. The LV endocardial border at the end-diastolic and end-systolic phases was traced automatically and manually to calculate the LV end-diastolic volume (LVEDV: $\mathrm{cm}^{3}$ ), LV end-systolic volume (LVESV: $\mathrm{cm}^{3}$ ) and LV ejection fraction (EF: \%). The endocardium and epicardium at the LV end-diastolic phase were also traced automatically and manually to calculate the LV mass (g) based on the myocardial tissue weight coefficient $(1.05 \mathrm{~g} / \mathrm{ml})$. LGE images of 6-8 slices from the area between the LV base and apex were obtained with the fast gradient 
$\mathbf{a}$

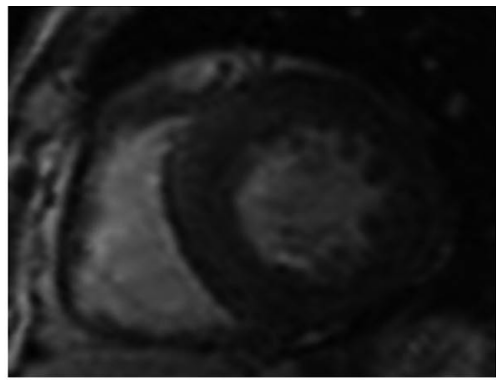

$\mathbf{C}$

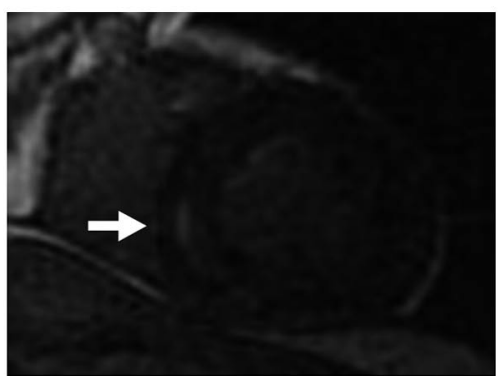

$\mathbf{b}$

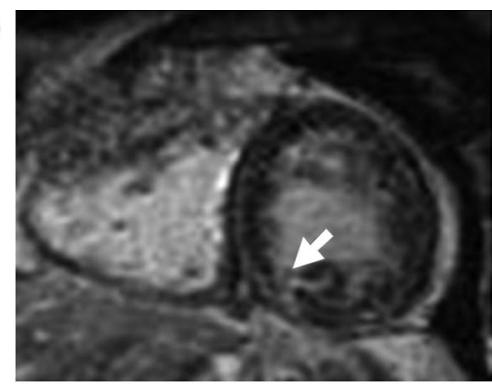

d

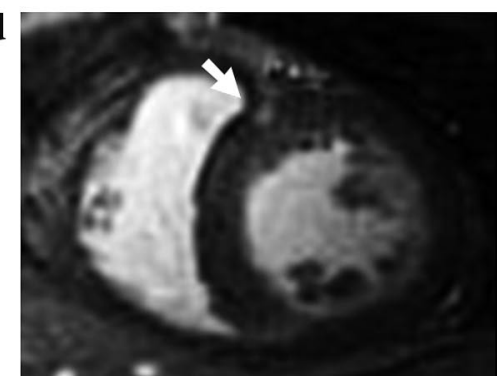

$\square I V S$

a (n)

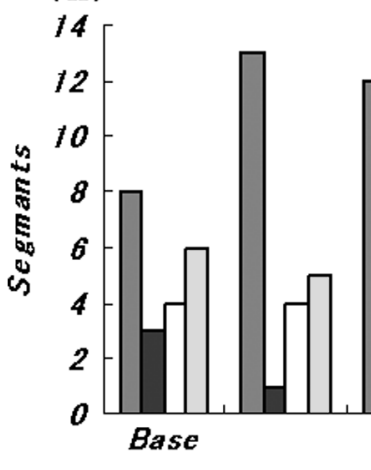

b

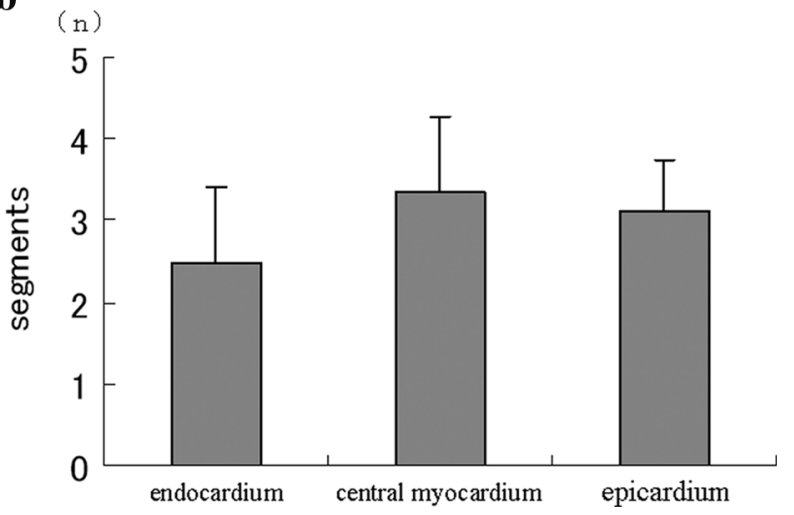

Figure 3. Late gadolinium enhancement patterns in dilated cardiomyopathy in short axis view. (a) Patient without late enhancement. (b-d) Patients with late enhancement. (b) The enhancement pattern is a diffuse midwall of intraventricular septum (IVS). (c) The enhancement pattern is the liner region of IVS. (d) The enhancement pattern is pathy region of IVS.

Figure 4. (a) The left ventricular myocardium was divided into 8 slices and 4 segments (anterior, lateral, posteroinferior and intraventricular septum (IVS) wall). Late gadolinium enhanced imaging (LGE) rate of base-mid IVS on left ventricular is higher than other segments. (b) There is no difference in the LGE rates of the endmyocardium, myocardium or epimyocardium.

field echo method (GE: TR $=7.1 \mathrm{~ms}, \mathrm{TE}=3.1 \mathrm{~ms}$, Flip angle $20^{\circ}$, TI $=150-250 \mathrm{~ms}$, FOV $38 \times 38 \mathrm{~cm}$, Matrix $256 \times 192$, Slice Thickness $=9 \mathrm{~mm}$, Gap $1 \mathrm{~mm}$ ) (Figure 1). Each slice was divided into segments of the anterior wall, lateral wall, inferior/posterior wall and septum, total areas were 493 of the localization of LGE. The distribution of contrast was evaluated in all slices with the left ventricle being divided into the endocardium, central myocardium, and epicardium, which were then further divided into 1,479 segments. The myocardial area and the contrast enhanced area were visu- ally extracted from each slice between the LV apex and base, and the areas (pixels) were calculated with Scion image software (version 4.02) on a Windows computer. Then the LV LGE rate was calculated using the following formula: LGE rate $=$ contrast enhanced area/LV myocardial area $\times 100$ (Figures 2a, b). ${ }^{11}$

LV Systolic and Diastolic Function on Echocardiography Echocardiography was performed within 2 weeks before or after the MRI. Echocardiographic measurements (2-dimen- 

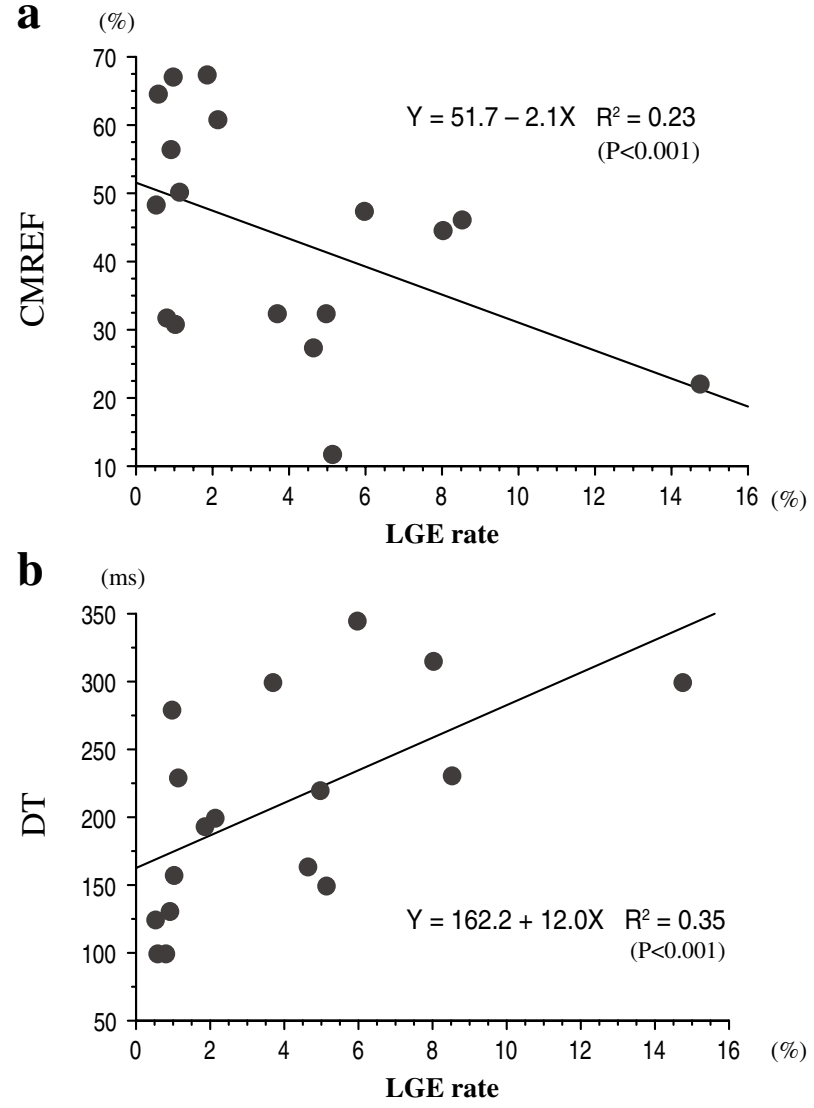

Figure 5. (a) Correlation of late gadolinium enhanced imaging (LGE) rate (\%) with DEMRI and ejection fraction (EF) with cine MRI. (b) Correlation of LGE rate (\%) and deceleration time (DT) with echocardiography.

sional and Doppler) were obtained by an observer who had no knowledge of the clinical and laboratory data of the patients. The LVEF was calculated by using the M-mode diameter method. Then the mitral inflow velocity was traced and the following variables were derived: peak velocity of early (E) filling, peak velocity of late (A) filling and deceleration time of the E wave velocity (DT: ms). Correlations were investigated between LVEDV, LVESV, LV mass, EF on CMR (CMREF), and LGE rate obtained through cardiac MRI and the E/A ratio, DT, LV end-diastolic diameter (LVDd), and EF measured using 2-dimensional echocardiography (Echo EF).

\section{Statistical Analysis}

Results are expressed as the mean \pm standard deviation. Student's t-test for paired data was used to assess statistical differences. Pearson's product moment correlation and regression analysis was used to assess the relations between the LGE rate (\%) and CMREF or DT. All analyses were performed with Stat View software (Version 5.0). A P value $<0.05$ was considered statistically significant.

\section{Results}

LGE

Echocardiography demonstrated that the Echo EF was $51.9 \pm 13.8 \%$ and LVDd was $62.9 \pm 8.2 \mathrm{~mm}$, suggesting that cardiac function in the chronic stage of DCM was relatively good in these patients (Table). IR-LGE at $20 \mathrm{~min}$ after infusion of Gd-DTPA was positive in 15 out of 17 patients $(88.2 \%)$. The LV segment contrast rate (LGE rate) was $3.84 \pm 3.9(0.54-14.7 \%)$. There were 3 patterns of LV contrast, which were patchy, linear and diffuse (Figures 3b-d). In 493 segments of slices between the base and apex, the segments with contrast enhancement included 57 (11.6\%) in the septum, $15(3 \%)$ in the anterior wall, $18(3.7 \%)$ in the lateral wall and $37(7.5 \%)$ in the inferior/posterior wall, indicating a high rate of contrast enhancement in the LV septal region (Figure 4a). The LV myocardium was divided into endocardial, central and epicardial layers, which were then divided into 1,479 segments. Contrast enhancement was observed in 37 endocardial segments $(2.5 \%), 55$ central segments $(3.7 \%)$ and 51 epicardial segments $(3.5 \%)$. There was no difference in the regions of contrast between the endocardial, central and epicardial layers (Figure $\mathbf{4 b}$ ).

\section{Correlations Between MRI and Echocardiography}

Automatic processing of cine MRI data was used to obtain the LV base and apex. The mean LVEDV was $169.5 \pm 55.0$ (91.5-244.2) $\mathrm{cm}^{3}$, the mean LVESV was 93.2 $\pm 46.2(33.9-$ 168.6) $\mathrm{cm}^{3}$, the mean CMREF was 43.8 $16.6(11.8-67.4)$ $\%$, and the mean LV mass was $135.0 \pm 40.0(93.6-247.3) \mathrm{g}$ (Table).

Correlations between LVDd, Echo EF and DT obtained using echocardiography and cardiac MRI were as follows: there was a correlation between LVEDV obtained by MRI and LVDd obtained using echocardiography $(\mathrm{Y}=-84.1+$ $\left.4.0 \mathrm{X}, \mathrm{R}^{2}=0.36, \mathrm{P}<0.001\right)$. There was a good correlation between CMREF and Echo EF $\left(\mathrm{Y}=-8.1+99.9 \mathrm{X}, \mathrm{R}^{2}=0.69\right.$, $\mathrm{P}<0.001)$

\section{LGE Rate, CMREF and LV Diastolic Function}

There was a correlation between CMREF and the LGE rate $\left(\mathrm{Y}=51.7-2.1 \mathrm{X}, \mathrm{R}^{2}=0.23, \mathrm{P}<0.001\right)$. There was also a correlation between DT or the E/A ratio obtained by echocardiography and the LGE rate $\left(\mathrm{Y}=162.2+12.0 \mathrm{X}, \mathrm{R}^{2}=0.35\right.$, $\mathrm{P}<0.001)$ (Figures 5a, b).

\section{Discussion}

It has been reported that MRI can provide information about tissues through abnormalities of SI or successive changes of SI due to contrast enhancement T1-weighted images. ${ }^{1-3}$ In recent years, investigation of LGE in DCM patients, who have relatively thin myocardium, as well as in patients with myocardial infarction and HCM, has revealed various imaging patterns..$^{5-8,15,16}$ In the present study, the LV pattern of LGE on cardiac MRI and correlations between the LGE rate and LV systolic function or LV diastolic function were investigated.

\section{Patterns of LGE}

In $88.2 \%$ (15 of 17 patients) with DCM, LV IR-LGE was observed, which was a high prevalence. However, some papers reported in 2003-2008 that the LGE positive rate in DCM patients differed significantly from $12 \%$ to $41 \%$, depending on the medical facility. ${ }^{6-8,17}$ All facilities used 1.5T MRI, but the Gd-DTPA concentration was 0.1$0.2 \mathrm{mmol} / \mathrm{kg}$, delayed imaging time $10-15 \mathrm{~min}$, slice thickness $7-10 \mathrm{~mm}$ and gap $0-3 \mathrm{~mm}$. LGE was obtained from different TE/TR, flip angles or inversion times. Under our hospital's imaging conditions, the mean LGE rate was $3.84 \pm 3.9 \%$, but patient dependent differences ranged from 
$0.54 \%$ to $14.7 \%$, and DCM patients appeared to be in different stages. In this study, the positive LGE rate was a high $88 \%$ using a GE 1.5 T MRI scanner, 9-mm slice thickness, Gd-DTPA dose of $0.15 \mathrm{mmol} / \mathrm{kg}$, and time delay of $20 \mathrm{~min}$.

The positive LGE rate is considered to vary depending on the imaging parameters, the volume of contrast medium injected, time delay, period since the onset of heart failure symptoms, duration of oral drug treatment and extent of myocardial damage as indicated by the severity of the patient's symptoms. The pattern of myocardial fibrosis in patients with DCM differs from that in patients with acute myocardial infarction. Since there are scattered fibrotic microlesions and the patterns show considerable diversity, the positive LGE rate also varies depending on differences in the criteria used to define LGE as positive. This study was performed with a contrast medium dose of $0.15 \mathrm{mmol} / \mathrm{kg}$ and was based on the SCMR protocol. However, the time delay was set at $20 \mathrm{~min}$ (vs $10 \mathrm{~min}$ in the SCMR protocol) and it is possible that the SNR was lower because of the long time delay in comparison with previous studies. Therefore a further study using the time delay specified in this protocol may be required. ${ }^{18}$

However, it is also possible that the positive LGE rate was high because the patients evaluated as positive included DCM patients with microlesions and a fibrotic patterns, as well as because of differences in the severity and the clinical course of each patient. ${ }^{6-8,17}$

Myocardial fibrosis and degeneration appeared to progress at different rates. There were 3 patterns of LV contrast, which were: (1) patchy; (2) linear; and (3) diffuse. Unlike the imaging patterns in acute myocardial infarction, there were various contrast patterns of the LV myocardium between the base and apex. ${ }^{6}$ The segments of the left ventricle that showed contrast enhancement included 57 $(11.6 \%)$ in the septum, $15(3 \%)$ in the anterior wall, 18 $(3.7 \%)$ in the lateral wall and $37(7.5 \%)$ in the inferior/ posterior wall. There were more enhanced septal segments between the base and the mid portion of the heart than in the lateral segments of the myocardium. There was no difference of contrast distribution among the endocardium, central layer and epicardium, as reported previously. Interstitial fibrosis has been reported as a pathological finding of DCM. ${ }^{6-8,13,19}$ Various patterns of fibrosis were revealed through LGE, suggesting that cardiac MRI is a possible modality for evaluating LV fibrosis in DCM. ${ }^{6-8}$ However, MRI might not be able to detect minimal fibrosis and such patients may have a negative LGE pattern.

\section{Cardiac MRI vs Echocardiography}

Cine MRI demonstrated that the mean LVEDV was $169.5 \pm$ $55.0 \mathrm{~cm}^{3}$, the mean LVESV was $93.2 \pm 46.2 \mathrm{~cm}^{3}$, the mean CMREF was $43.8 \pm 16.6 \%$ and the mean LV mass was $135.0 \pm 40.0 \mathrm{~g}$. There was a correlation between LVEDV (CMR) and LVDd (echocardiography), as well as between CMREF and Echo EF, indicating that volume data obtained using cardiac MRI were more accurate than those from echocardiography, as previously reported.

\section{LGE Rate, CMREF and LV Diastolic Function}

Bohl reported on the correlation between late gadolinium hyperenhancement and LVEF and a similar result was provided in our study. ${ }^{17}$ This finding may suggest that the decrease of CMREF is due to progressive LV fibrosis, as demonstrated by the LGE rate. Thus, cardiac function and prognosis may be predicted from the LGE rate. ${ }^{20-23}$

There was a good correlation between the LGE rate and DT, suggesting that the extent of LV fibrosis reflects LV diastolic dysfunction, which occurs earlier than systolic dysfunction. It seems that the amount of interstitial fibrosis is more important than hypertrophy of the LV myocardium because it influences myocardial stiffness. It has been reported that collagen accumulates along with progression of diastolic dysfunction and that collagen changes qualitatively, with an increased type I/III ratio and more crosslinking. ${ }^{13}$ It was established by the pathological studies of Kim et al that LGE on cardiac MRI indicates myocardial fibrosis. It seems possible to detect fibrosis, which means collagen accumulation, even though the quality of fibrosis cannot be assessed. ${ }^{23}$ In the present study, correlations were found between the LGE rate and decreased LV systolic function, as well as diastolic dysfunction. It is possible that the LGE pattern and LGE rate reflect LV fibrosis and can predict the response of LV systolic function and LV diastolic function to oral medication with $\beta$-blockers, ACE-I, and ARB. ${ }^{24}$

\section{Study Limitations}

A 1-mm slice gap was employed in the present study. It would be desirable to have no slice interval in order to collect more accurate data. Contrast enhancement was determined visually based on comparison with acute myocardial infarction. Automatic establishment of a contrast threshold may be necessary in order to better determine the contrast enhancement of the left ventricle.

This study was performed with a contrast medium dose of $0.15 \mathrm{mmol} / \mathrm{kg}$ and was based on the SCMR protocol. However, the time delay was set at $20 \mathrm{~min}$ (vs $10 \mathrm{~min}$ in the SCMR protocol), so a further study using the time delay specified in this protocol may be required. ${ }^{18}$

\section{Conclusion}

In patients with myocardial diseases such as AMI, HCM and DCM, clarification of myocardial fibrosis and myocardial disorders using MRI or especially LGE is recommended. In DCM patients, high contrast positive rates were found but myocardial contrast rates differed by patient. Contrast effects were often seen in the septum and did not differ for the endocardium, tunica media and epicardium. This suggested that DCM patients were classified by LGE. The LGE correlated with CMREF and LV diastolic function. Since LGE reflects different LV conditions, this appeared highly useful.

\section{Reference}

1. Farmer D, Higgins CB, Yee E, Lipton M, Wahr D, Ports T. Tissue characterization by magnetic resonance imaging in hypertrophic cardiomyopathy. Am J Cardiology 1985; 55: 230-232.

2. Nanjo S, Yamazaki J, Yoshikawa K, Miura M, Seno A. Efficacy of contrast-enhanced MR imaging in cardiomyopathy: An experimental study using Bio14.6 hamsters. Acad Radiol 2002; 9: 1139-1147.

3. Nanjo S, Yamazaki J, Yoshikawa K, Ishii T, Togane Y. Carvedilol prevents myocardial fibrosis in hamster. Int Heart J 2006; 47: 607 616.

4. Kim RJ, Fieno DS, Parrish TB, Harris K, Chen EL, Simonetti O, et al. Relationship of MRI delayed contrast enhancement to irreversible injury, infarct age, and contractile function. Circulation 1999; 100: 1992-2002.

5. Kim RJ, Wu E, Rafael A, Chen EL, Parker MA, Simonetti O, et al. The use of contrast-enhanced magnetic resonance imaging to identify reversible myocardial dysfunction. $N$ Engl J Med 2000; 343: 
$1445-1453$

6. McCrohon JA, Moon JC, Prasad SK, McKenna WJ, Lorenz CH, Coats AJ, et al. Differentiation of heart failure related to dilated cardiomyopathy and coronary artery disease using gadolinium enhanced cardiovascular magnetic resonance. Circulation 2003; 108: 54-59.

7. Assomull RG, Prasad SK, Lyne J, Smith G, Burman ED, Khan M, et al. Cardiovascular magnetic resonance, fibrosis, and prognosis in dilated cardiomyopathy. J Am Coll Cardiol 2006; 48: 1977-1985.

8. Matoh F, Satoh H, Shiraki K, Saitoh T, Urushida T, Katoh H, et al Usefulness of delayed enhancement magnetic resonance imaging to differentiate dilated phase of hypertrophic cardiomyopathy and dilated cardiomyopathy. J Card Fail 2007; 13: 372-379.

9. Doi R, Masuyama T, Yamamoto K, Doi Y, Mano T, Sakata Y, et al. Development of different phenotypes of hypertensive heart failure: Systolic versus diastolic failure in Dahl salt-sensitive rats. Hypertension 2000; 18: $111-120$.

10. Redfield MM, Jacobsen SJ, Burnett JC Jr, Mahoney DW, Bailey KR, Rodeheffer RJ. Burden of systolic and diastolic ventricular dysfunction in the community: Appreciating the scope of the heart failure epidemic. JAMA 2003; 289: 194-202.

11. Motoyasu M, Kurita T, Onishi K, Uemura S, Tanigawa T, Okinaka $\mathrm{T}$, et al. Correlation between late gadolinium enhancement and diastolic function in hypertrophic cardiomyopathy assessed by magnetic resonance imaging. Circ J 2008; 72: 378-383.

12. WHO/ISFC task force on the definition and classification of cardiomyopathies. Circulation 1996; 93: 841-842.

13. Richardson P, McKenna W, Bristow M, Maisch B, Mautner B, O'Connell J, et al. Report of the 1995 World Health Organization/ International Society and Federation of Cardiology Task Force on the definition and classification of cardiomyopathies. Circulation 1996; 93: $841-842$

14. Mahrholdt H, Goedecke C, Wagner A, Meinhardt G, Athanasiadis A, Vogelsberg $\mathrm{H}$, et al. Cardiovascular magnetic resonance assessment of human myocarditis: A comparison to histology and molecular pathology. Circulation 2004; 109: 1250-1258.

15. Teraoka K, Hirano M, Ookubo H, Sasaki K, Katsuyama H, Amino $\mathrm{M}$, et al. Delayed contrast enhancement of MRI in hypertrophic cardiomyopathy. Magn Reson Imaging 2004; 22: 155-161.

16. Choi DS, Ha JW, Choi B, Yang WI, Choi EY, Rim SJ, et al. Extent of late gadolinium enhancement in cardiovascular magnetic resonance and its relation with left ventricular diastolic function in patients with hypertrophic cardiomyopathy. Circ J 2008; 72: 1449-1453.

17. Bohl S, Wassmuth R, Abdel-Aty H, Rudolph A, Messroghli D, Dietz $\mathrm{R}$, et al. Delayed enhancement cardiac magnetic resonance imaging reveals typical patterns of myocardial injury in patients with various forms of non-ischemic heart disease. Int J Cardiovasc Imaging 2008; 24: 597-607.

18. Kramer CM, Barkhausen J, Flamm SD, Kim RJ, Nagel E. Standardized cardiovascular magnetic resonance imaging (CMR) protocols, society for cardiovascular magnetic resonance: board of trustees task force on standardized protocols. Journal of CMR 2008; 10:35 doi: 10.1186/1532-429X-10-35.

19. de Leeuw N, Ruiter DJ, Balk AH, de Jonge N, Melchers WJ, Galama JM. Histopathologic findings in explanted heart tissue from patients with end-stage idiopathic dilated cardiomyopathy. Transpl Int 2001; 14: 299-306.

20. Yan AT, Shayne AJ, Brown KA, Gupta SN, Chan CW, Luu TM, et al. Characterization of the peri-infarct zone by contrast-enhanced cardiac magnetic resonance imaging is a powerful predictor of post-myocardial infarction mortality. Circulation 2006; 114: 32-39.

21. Kwong RY, Chan AK, Brown KA, Chan CW, Reynolds HG, Tsang S, et al. Impact of unrecognized myocardial scar detected by cardiac magnetic resonance imaging on event-free survival in patients presenting with signs or symptoms of coronary artery disease. Circulation 2006; 113: $2733-2743$.

22. Assomull RG, Prasad SK, Lyne J, Smith G, Burman ED, Khan M, et al. Cardiovascular magnetic resonance, fibrosis, and prognosis in dilated cardiomyopathy. J Am Coll Cardiol 2006; 48: 1977-1985

23. Yamamoto $K$, Masuyama T, Sakata $Y$, Nishikawa N, Mano T, Yoshida J, et al. Myocardial stiffness is determined by ventricular fibrosis, but not by compensatory or excessive hypertrophy in hypertensive heart. Cardiovasc Res 2002; 55: 76-82.

24. Izawa $\mathrm{H}$, Murohara $\mathrm{T}$, Nagata $\mathrm{K}$, Isobe $\mathrm{S}$, Asano $\mathrm{H}$, Amano $\mathrm{T}$, et al. Mineralocorticoid receptor antagonism ameliorates left ventricular diastolic dysfunction and myocardial fibrosis in mildly symptomatic patients with idiopathic dilated cardiomyopathy: A pilot study. Circulation 2005; 112: 2940-2945. 